\title{
FDA Boxed Warning for Montelukast: Impact on Adult Severe Asthmatics?
}

\section{Dear Editor,}

Montelukast, a leukotriene receptor antagonist, was first approved by the US Food and Drug Administration (FDA) for treatment of asthma and allergies in 1998. It is approved for long-term treatment of asthma in adults and children 1 year and older; prevention of exercise-induced asthma in patients 6 years and older; for seasonal allergic rhinitis in patients 2 years and older; and perennial allergic rhinitis in those 6 months and older. On 4 March 2020, FDA announced the requirement for Boxed Warning about serious mental health side effects (including suicidal thoughts or actions) with use of montelukast and advises restricting use for allergic rhinitis, especially if there are other treatment alternatives. ${ }^{1}$ For patients with asthma, FDA recommends consideration of benefits and risks in prescription of montelukast. The FDA recommendation is based on case reports of a wide variety of mental health effects occurring during and even after stopping montelukast. However, sentinel and prior observational studies in asthmatic patients aged $\geq 6$ years old, did not find an increased risk of mental health side effects in comparison to inhaled corticosteroids. ${ }^{1-4}$ There are limited reports on mental health effects of montelukast in the adult patients with severe asthma. To fill this gap, we compared psychological impairment and the Hospital Anxiety and Depression scale (HADS) scores of patients prescribed with montelukast and those without, in the Singapore General Hospital registry of severe asthma patients.

Patient with severe asthma receiving step 4 or 5 of Global Initiative for Asthma (GINA) 2019 treatment ladder were recruited into our severe asthma registry. ${ }^{5}$ Asthma was defined as the presence of variable symptoms and expiratory airflow limitation according to the GINA guidelines. ${ }^{5}$ HADS score was obtained at regular clinic visit. The use of montelukast, duration on montelukast, and HADS scores during montelukast use were reviewed retrospectively. The diagnosis of anxiety and depression was based on DSM-V criteria by a registered psychiatrist. This study was approved by the Institutional Review Boards (IRBs) under IRB: 2010/810/C. Written informed consent was obtained from participants.

A total of 199 severe asthma patients with available HADS scores were included in the final analysis. The median age of the recruited cohort was 51 years (interquartile range [IQR] 38-62 years), median body mass index of $25.8 \mathrm{~kg} / \mathrm{m}^{2}$ (IQR $22.4-29.8 \mathrm{~kg} / \mathrm{m}^{2}$ ) and $62.8 \%$ were female $(\mathrm{n}=125)$. Of the 199 patients, $83(41.7 \%)$ were on montelukast at the time of HADS score assessment. The median days on montelukast was 2,046 (IQR 628-3,090). There are 11 patients with existing diagnosis of anxiety and 11 with depression. On multivariate analysis (after adjustment for age, gender, body mass index, race and asthma control test score), there is no association between montelukast and anxiety or depression symptoms (HADS $\geq 8$ ) (adjusted odds ratio (OR) $1.09,96 \%$ confidence interval [CI] $0.56-2.13, P=0.79$ ), or presence or absence of psychiatric diagnosis of anxiety or depression (adjusted OR $3.25,95 \%$ CI $0.87-14.16, P=0.09$ ). There is only 1 patient with a previous suicide attempt but she had a pre-existing diagnosis of depression prior to commencement of montelukast.

Evidence from our real-world data does not seem to suggest an association between montelukast use and presence or absence of psychological impairment in the adult severe asthma population. Montelukast is an alternative treatment for asthma from step 1 to 5 in GINA treatment ladder and frequently used as an add-on in patients on step 4 and 5 for added control. ${ }^{5}$ While our results may be reassuring, we acknowledge limitations in observational studies: causal relationship cannot be proven and the presence of confounders (e.g. severity of disease or allergic comorbidities). Nevertheless, our registry finding adds to the body of evidence that montelukast is safe, at least in the adult asthma population, and there is no urgent need to eliminate it from our medication list. Future studies evaluating the effect of montelukast in adult severe asthma patients are required to further validate our findings. 


\section{REFERENCES}

1. FDA Drug Safety Communication, 13 March 2020. Available at: https://www.fda.gov/drugs/drug-safety-and-availability/fda-requiresboxed-warning-about-serious-mental-health-side-effects-asthma-andallergy-drug\#5e649af17ddd1. Accessed on 7 September 2020.

2. Glockler-Lauf SD, Finkelstein Y, Zhu J, et al. Montelukast and Neuropsychiatric Events in Children with Asthma: A Nested CaseControl Study. J Pediatr 2019;209:176-82.e4.

3. Law SWY, Wong AYS, Anand S, et al. Neuropsychiatric Events Associated with Leukotriene-Modifying Agents: A Systematic Review. Drug Saf 2018;41:253-65.

4. Benard B, Bastien V, Vinet B, et al. Neuropsychiatric adverse drug reactions in children initiated on montelukast in real-life practice. Eur Respir J 2017;50:1700148.

5. Global Initiative for Asthma. Global Management and Prevention, 2014. Available at: www.ginasthma.org. Accessed on 7 September 2020.
Pei Yee Tiew, ${ }^{1-3} M B B S, M R C P$, Karen Li Leng Tan, ${ }_{B N}$, Mariko Siyue $\underline{\mathrm{Koh}},{ }^{1-3}$ MBBS, MRCP

${ }^{1}$ Department of Respiratory and Critical Care Medicine, Singapore General Hospital, Singapore

${ }^{2}$ Lee Kong Chian School of Medicine, Nanyang Technological University, Singapore

${ }^{3}$ Duke-NUS Medical School, Singapore

Address for Correspondence: Dr Pei Yee Tiew, Department of Respiratory and Critical Care Medicine, Singapore General Hospital, Outram Road, Singapore 169608. Email: tiew.pei.yee@singhealth.com.sg 